\title{
Consumo de Energia e Crescimento Econômico: uma Análise do Brasil no período 1970-2009
}

\section{Energy Consumption and Economic Growth: an Analysis of Brazil in the period 1970-2009}

\author{
Amir Borges Ferreira Neto* \\ Wilson Luiz Rotatori Corrêa** \\ Fernando Salgueiro Perobelli* * *
}

Resumo: O presente artigo se propõe a contribuir para a literatura que versa sobre crescimento e consumo de energia ao investigar a direção de causalidade, por meio de um modelo empírico, para consumo de energia e crescimento econômico. Para atingir tal objetivo, o artigo faz uso da metodologia de séries temporais e usa dados de crescimento e do PIB do período brasileiro de 1970 a 2009. Os resultados mostram que a direção de causalidade parece ser do consumo de energia total para o PIB, embora a causalidade bidirecional não possa ser descartada, e que, ao se considerar a renda das famílias e o consumo de eletricidade, por sua vez, a direção de causalidade é bem definida da renda bruta disponível para o consumo residencial.

Palavras-chave: Energia. Crescimento econômico. Séries temporais. Brasil.

\begin{abstract}
This article aims to contribute to the literature that deals with growth and energy consumption investigating the direction of causality by means of an empirical model for energy consumption and economic growth. To achieve this goal the article makes use of time series methodology and uses data from Brazil in the period of 1970 to 2009. The results show that the direction of causality seems to be from total energy consumption to GDP, although a bidirectional causality cannot be discarded. Also, when considering household income and electricity consumption, the evidence points to a well-defined direction of causality from gross disposable income to household electricity consumption.
\end{abstract}

Keywords: Energy. Economic growth. Time series. Brazil.

JEL Classification: C32; Q43.

\footnotetext{
* Doutorando em Economia pelo Departamento de Economia da West Virginia University e Graduate Research Assistant no Regional Research Institute da West Virginia University. E-mail: amneto@mix.wvu.edu

* Doutor em Economia. Professor do Departamento de Economia e do Programa de PósGraduação em Economia Aplicada (PPGE) da Universidade Federal de Juiz de Fora (UFJF). E-mail: wilson.rotatori@ufff.edu.br

*** Doutor em Economia. Professor do Departamento de Economia e do Programa de PósGraduação em Economia Aplicada (PPGE) da Universidade Federal de Juiz de Fora (UFJF). Bolsista de produtividade CNPq. E-mail: fernando.perobelli@ufjf.edu.br. O autor agradece à Fapemig e ao CNPq pelo apoio financeiro para a realização desta pesquisa.
} 


\section{Introdução}

O crescimento econômico de um país ou uma região está intimamente ligado à disponibilidade de insumos para o processo produtivo, dentre eles destacam-se a terra, o trabalho, o capital e a energia. Para que haja um desenvolvimento sustentável, é preciso que uma determinada região possua um planejamento de como o fornecimento desses recursos será feito no longo prazo para que não haja excessos ou faltas. Por meio da Tabela 1, é possível fazer uma análise do comportamento histórico da evolução recente do consumo de energia elétrica no Brasil.

Assim como outros países em desenvolvimento, o Brasil passa por um momento de aumento na demanda por energia. Por exemplo, entre 1990 e 2009 a taxa de crescimento médio do consumo de energia no país foi de 3,41\% ao ano, enquanto que o PIB real cresceu 3,49\% por ano, em média, para o mesmo período. O consumo de energia per capita variou de 1,485 KWh, em 1990, para 2,225 KWh em 2009.

Tabela 1 - Brasil: consumo de energia elétrica total

\begin{tabular}{l|c|c|c|c}
\hline Ano & $\begin{array}{c}\text { Consumo de } \\
\text { eletricidade total } \\
\text { (103 GWh) }\end{array}$ & $\begin{array}{c}\text { Consumo de } \\
\text { eletricidade } \\
\text { per capita }\end{array}$ & $\begin{array}{c}\text { Taxa de } \\
\text { variação } \\
\text { anual (\%) }\end{array}$ & $\begin{array}{c}\text { Taxa de } \\
\text { variação } \\
\text { acumulada } \\
\text { (\%) }\end{array}$ \\
\hline 1990 & 217657 & 1,485 & - & - \\
1991 & 225372 & 1,512 & 1,035 & 1,035 \\
1992 & 230472 & 1,521 & 1,023 & 1,058 \\
1993 & 241167 & 1,566 & 1,046 & 1,104 \\
1994 & 249793 & 1,597 & 1,036 & 1,140 \\
1995 & 264805 & 1,667 & 1,060 & 1,200 \\
1996 & 277685 & 1,721 & 1,049 & 1,249 \\
1997 & 294689 & 1,799 & 1,061 & 1,310 \\
1998 & 307030 & 1,847 & 1,042 & 1,352 \\
1999 & 315753 & 1,871 & 1,028 & 1,381 \\
2000 & 331638 & 1,936 & 1,050 & 1,431 \\
2001 & 309729 & 1,782 & 0,934 & 1,365 \\
2002 & 324365 & 1,840 & 1,047 & 1,412 \\
2003 & 342213 & 1,915 & 1,055 & 1,467 \\
2004 & 359945 & 1,987 & 1,052 & 1,519 \\
2005 & 375193 & 2,046 & 1,042 & 1,561 \\
\hline & & & & continua...
\end{tabular}




\begin{tabular}{l|c|c|c|c} 
conclusão. & $\begin{array}{c}\text { Consumo de } \\
\text { eletricidade total } \\
\text { (103 } \mathbf{~ G W h ) ~}\end{array}$ & $\begin{array}{c}\text { Consumo de } \\
\text { eletricidade } \\
\text { per capita }\end{array}$ & $\begin{array}{c}\text { Taxa de } \\
\text { variação } \\
\text { anual (\%) }\end{array}$ & $\begin{array}{c}\text { Taxa de } \\
\text { variação } \\
\text { acumulada } \\
\text { (\%) }\end{array}$ \\
\hline 2006 & 389950 & 2,101 & 1,039 & 1,601 \\
2007 & 412130 & 2,196 & 1,057 & 1,657 \\
2008 & 428250 & 2,259 & 1,039 & 1,697 \\
2009 & 426029 & 2,225 & 0,995 & 1,691 \\
\hline
\end{tabular}

Fonte: Elaboração própria a partir do Balanço Energético e IBGE.

Um exemplo recente de escassez de insumo produtivo é o da crise energética brasileira ocorrida nos anos de 2001 e 2002. Nesse período, o país teve um forte desequilíbrio entre a oferta de energia elétrica e a previsão de consumo, fato que se deu graças ao baixo nível observado nos reservatórios das hidrelétricas nas regiões Nordeste, Sudeste e Centro-Oeste. Pêgo-Filho et al. (2001) destacam três causas estruturais gerais para a crise: tempo de duração e falta de sincronia no processo de transição do modelo institucional baseado no Estado para o modelo no qual o setor privado é responsável pelo suprimento de eletricidade; aumento dos riscos envolvidos com a regulação; e falta de articulação na criação e implementação das reformas nos setores elétrico, petróleo e gás natural.

Embora a teoria econômica não estabeleça de forma explícita a relação entre consumo de energia e crescimento econômico, uma série de trabalhos empíricos têm sido realizados com o intuito de analisar tal relação. Desde o trabalho seminal de Kraft e Kraft (1978), diversos estudos têm como objeto a relação causal entre consumo de energia e crescimento econômico, seja por modelos bivariados (YANG, 2000; SOYTAS; SARI, 2003), seja por meio de modelos multivariados (GLASURE, 2002; HONDROYIANNISET; LOLOS; PAPAPETROU, 2002; GHALI; EL-SAKKA, 2004). Embora não haja ainda consenso tanto no que tange à existência e à direção da causalidade de Granger entre consumo de energia e crescimento econômico, Stern (1997) constata um ponto comum na literatura: energia é essencial para o processo produtivo.

A falta de consenso em relação à causalidade entre energia e produção de bens e serviços pode ser devido ao fato de que os países têm diferentes padrões de consumo de energia e às diferentes fontes de energia. Portanto, o uso de fontes diferentes de energia pode ter impactos variados sobre o produto da economia. Sari e Soytas (2004) e Yang (2000) fazem análises que levam em conta a relação entre consumo de energia desagregado por fontes de energia e renda. De acordo com Soytas e Sari (2007), a maioria dos trabalhos existentes na literatura, incluindo aqueles que empregam medidas desagregadas de energia, trata as questões em 
termos macro. Nesses trabalhos, a ligação entre as séries temporais de consumo de energia e PIB são analisadas. Entretanto, se os setores que são intensivos no consumo de energia não são aqueles que podem ser tomados como setor-chave da economia, tal relação pode não ser captada quando se trata da questão de forma agregada.

Outro fato que pode levar a resultados conflitantes, no que se refere aos resultados empíricos da relação entre consumo de energia e crescimento econômico, está centrado na variedade de abordagens e nos procedimentos de testes empregados para discutir tais questões. Os primeiros estudos que buscavam tratar dessa questão empregavam modelos log-linear estimado por mínimos quadrados ordinários sem levar em conta a natureza e as propriedades das séries temporais das variáveis escolhidas. Entretanto, a maioria das séries temporais de variáveis macroeconômicas apresentam algum tipo de não estacionariedade, como discutido em Granger e Newbold (1974) e Nelson e Plosser (1982). Portanto, ao não considerar tais questões, poder-se-ia incorrer em erros de mensuração nas relações entre crescimento econômico e consumo de energia (ASAFU-ADJAYE, 2000).

A direção da causalidade entre consumo de energia e crescimento econômico tem implicações de política significantes. Se, por exemplo, existir causalidade de Granger unidirecional da renda para o consumo de energia, isso pode indicar que políticas de conservação de energia podem ser implementadas com pequeno, ou, até mesmo, nenhum efeito sobre o crescimento econômico. Segundo Akarca e Long (1979), no caso de causalidade negativa do emprego para energia, pode ocorrer um aumento no emprego total se políticas de conservação de energia for implementadas. Por outro lado, se há causalidade unidirecional do consumo de energia em direção à renda, a redução no consumo de energia poderia ocasionar uma queda na renda ou no emprego. Os resultados de não causalidade em ambas as direções, ou seja, a hipótese de neutralidade pode implicar que políticas de conservação de energia não afetariam o crescimento econômico.

Embora exista uma extensa discussão na literatura, como em Glasure e Lee (1998), Stern (2000), Soytas e Sari (2003) e Oh e Lee (2004a, 2004b) a respeito da direção de causalidade entre consumo de energia e crescimento econômico especificamente para o caso brasileiro, não se tem conhecimento até o presente momento de uma análise desse tipo. Considerando a importância do tema para o crescimento econômico e a geração de empregos como discutido anteriormente, o presente artigo se propõe a investigar essa direção de causalidade por meio de um modelo empírico para consumo de energia e crescimento econômico. Para atingir esse objetivo, são utilizadas séries de tempo sobre o consumo de energia total no Brasil e o PIB brasileiro a partir de 1970. É importante destacar que, ao se falar em consumo de energia, visa-se uma análise essencialmente de demanda, de 
tal forma que os dados utilizados são os dados de consumo efetivo de energia, e não os de oferta ou previsão de demanda.

Uma análise complementar realizada neste trabalho, com a mesma abordagem, busca entender o efeito da renda no consumo residencial de eletricidade, principal fonte energética utilizada pelas famílias no Brasil. Dessa forma, são utilizados dados para o mesmo período do consumo de energia elétrica residencial e dados relativos à renda bruta disponível. Por avaliar ambos, energia total e energia elétrica residencial, decidiu-se restringir o período de análise a a partir de 1970, quando ambas as séries apresentam dados até 2009, último ano disponível.

Considerando que o foco central do presente trabalho é investigar a direção de causalidade entre crescimento e consumo de energia a partir de um modelo empírico, se omite uma discussão mais aprofundada sobre a metodologia a ser empregada. Em particular, para investigar a direção de causalidade entre consumo de energia e crescimento, utilizamos a abordagem de séries de tempo, em que são realizados testes para primeiro investigar a presença de raízes unitárias nas séries do consumo de energia, PIB e renda bruta disponível. A abordagem teórica para os testes de raiz unitária é extensamente discutida na literatura, como em Dickey e Fuller (1979, 1981), Perron (1989), Kwiatkowski et al. (1992), Elliot, Rothenberg e Stock (1996), Maddala e Kim (1998), NG e Perron (2001), Carrion-i-Silvestre et al. (2009), entre outros.

Num segundo momento, na definição do modelo empírico e realização dos testes de causalidade, utiliza-se a abordagem dos modelos autorregressivos vetoriais (VAR). O seu desenvolvimento teórico e implicações também são extensamente discutidos na literatura, sendo inicialmente proposto por Sims (1980) e sintetizado por Hamilton (1994), Canova (1995), Hendry (1995), Johansen (1996), Lutkhepol (2005) e Juselius (2006), entre outros. Desse modo, pelo mesmo motivo, se omite uma discussão aprofundada sobre a metodologia.

Este trabalho, além desta parte introdutória, está organizado em uma breve apresentação do banco de dados e discussão das propriedades estatísticas básicas das séries na segunda seção. Na terceira seção são discutidos os modelos empíricos e os resultados. Por fim, na quarta seção são apresentadas as conclusões finais e as perspectivas para novos estudos.

\section{Propriedades Estatísticas dos Dados}

Nesta seção serão apresentados os dados utilizados e os testes realizados no presente estudo. O objetivo é determinar as propriedades estatísticas e investigar a presença de não estacionariedade nas séries, procurando, dessa forma, definir a ordem de integração, bem como se outros tipos de não estacionariedade, como 
quebras estruturais, se mostram presentes. Todos os resultados foram gerados utilizando-se os softwares Rats versão 7 e Cats versão 2.

As Figuras 1 e 3 apresentam as séries do logaritmo da energia total consumida (LET) e do PIB ${ }^{1}$ (LPIB) do Brasil entre 1970 e 2009, enquanto que as Figuras 2 e 4 apresentam as séries tomadas em primeira diferença.

Figura 1 - Log do Consumo Total de Energia

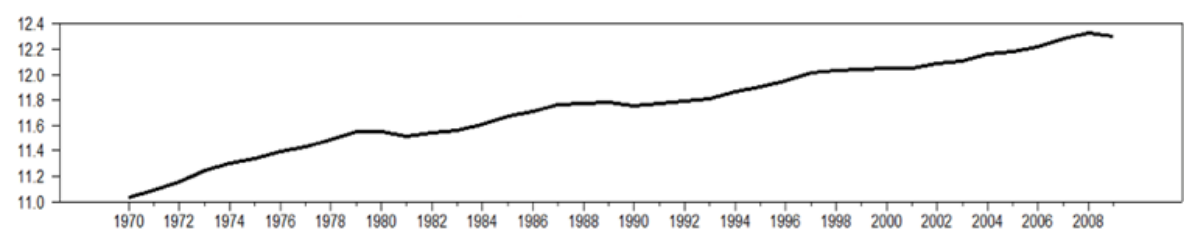

Fonte: Elaboração própria dos autores.

Figura 2 - Primeira Diferença do Log do Consumo Total de Energia

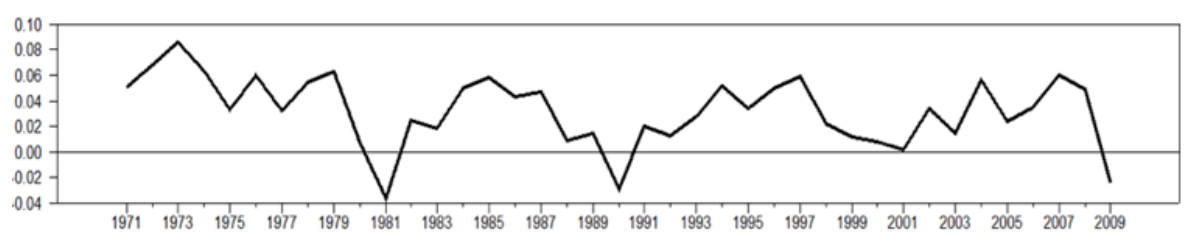

Fonte: Elaboração própria dos autores.

Figura 3 - Log do PIB

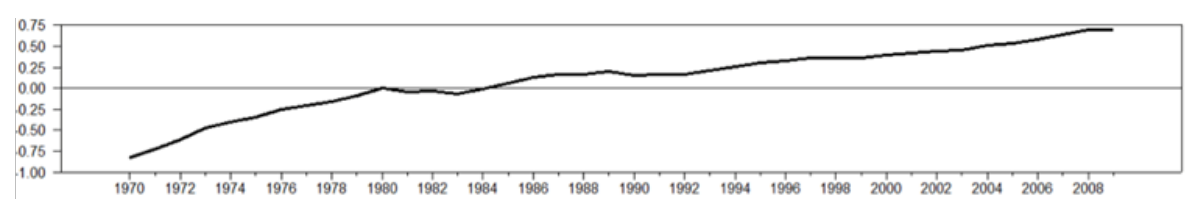

Fonte: Elaboração própria dos autores.

Figura 4 - Primeira Diferença do Log do PIB

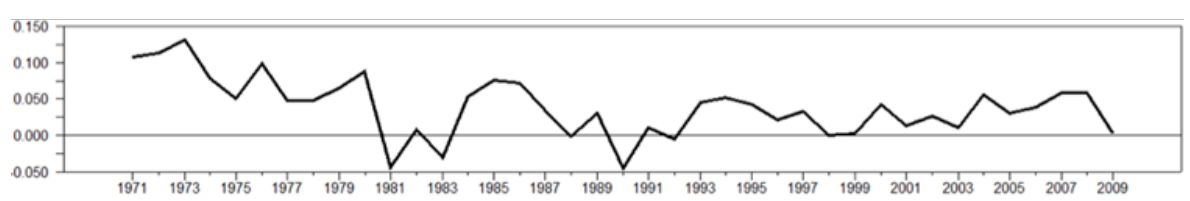

Fonte: Elaboração própria dos autores.

$\overline{1}$ Índice construído no Ipeadata utilizando-se o deflator implícito do PIB com 1980 = 100, sendo que o mesmo foi utilizado para a série de renda bruta disponível a ser apresentada posteriormente. 
Uma característica distintiva do comportamento das séries em nível é a existência de uma tendência de crescimento ao longo do tempo, o que conduz à investigação da hipótese das variáveis seguirem um processo de tendência estacionária, em que, apesar da média da série não ser constante no período amostral, a variável é passível de ser prevista, na medida em que possa ser considerada estacionária em torno de uma tendência determinística.

De outro modo, as variáveis em primeira diferença apresentam um comportamento estacionário em torno de uma média de longo prazo, o que leva a testar a hipótese das séries serem integradas de ordem 1, ou I(1), caso em que seguiriam um processo estacionário em diferenças. Nesse sentido, foi formulada a hipótese para os testes de raiz unitária. Inclui-se um termo determinístico procurando identificar se as séries apresentam um comportamento de um processo estacionário em torno de uma tendência, ou de um processo estacionário em diferenças.

As Tabelas 2 e 3 apresentam os resultados dos testes de raízes unitárias, para séries de energia total e PIB, respectivamente, sendo apresentadas as defasagens utilizadas nos testes, a estatística de cada teste e os valores críticos de 1\% e 5\%. Considerando os problemas existentes com o tamanho e poder dos testes de raiz unitária extensamente discutidos na literatura, ${ }^{2}$ optou-se pela realização de diferentes testes, a saber, Dickey-Fuller aumentado (ADF), Phillips Perron (PP), Elliot Rothenberg e Stock (ERS) nas formas PT (Point Optimal) e DF-GLS (Dickey-Fuller-Generalized Least Square) Kwiatkowski, Phillips, Shmidt e Shin (KPSS) e, por fim, NG e Perron. O objetivo é utilizar-se inicialmente dos resultados dos testes de ADF e PP em comparação com as correções propostas nos testes ERS e NG e Perron, bem como estabelecer uma análise confirmatória com o teste KPSS, cuja hipótese nula é de que as séries sejam I(0) contra I(1) nos demais testes.

2 Para uma discussão e sumário dos principais resultados a respeito do tema, ver Maddala e Kim (1998). 
Tabela 2 - Testes de raiz unitária para a série de energia total

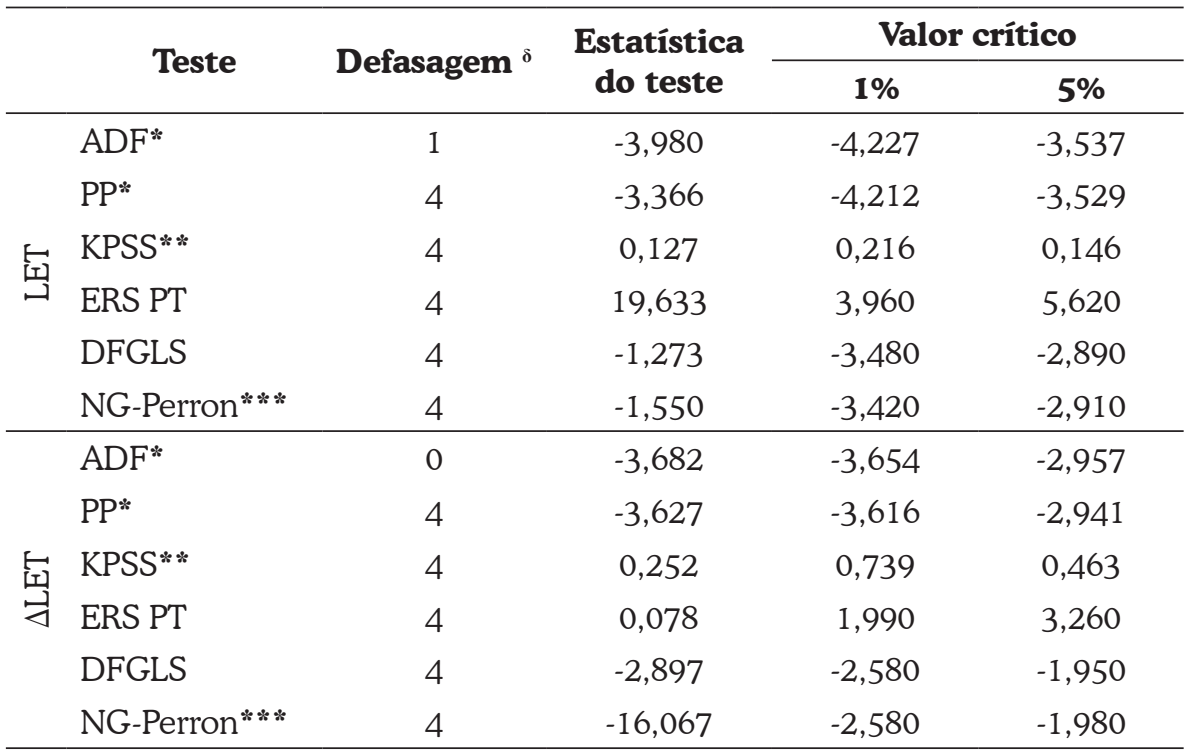

Fonte: Elaboração própria.

Nota: $\delta$ Defasagem minimiza o critério de Schwartz; " tabela MacKinnon (1996); ** tabela Kwiatkowski-Phillips-Schmidt-Shin (1992); *** tabela Perron (2001).

Tabela 3 - Testes de raiz unitária para a série do PIB

\begin{tabular}{|c|c|c|c|c|c|}
\hline & \multirow{2}{*}{ Teste } & \multirow{2}{*}{ Defasagem $^{\delta}$} & \multirow{2}{*}{$\begin{array}{l}\text { Estatística } \\
\text { do teste }\end{array}$} & \multicolumn{2}{|c|}{ Valor crítico } \\
\hline & & & & $1 \%$ & $5 \%$ \\
\hline \multirow{6}{*}{$\stackrel{\varphi}{9}$} & $\mathrm{ADF}^{*}$ & 0 & $-4,580$ & $-4,227$ & $-3,537$ \\
\hline & $\mathrm{PP}^{*}$ & 4 & $-4,713$ & $-4,212$ & $-3,529$ \\
\hline & KPSS** & 4 & 0,168 & 0,216 & 0,146 \\
\hline & ERS PT & 4 & 31,467 & 3,960 & 5,620 \\
\hline & DFGLS & 4 & $-1,181$ & $-3,480$ & $-2,890$ \\
\hline & NG-Perron*** & 4 & $-1,270$ & $-3,420$ & $-2,910$ \\
\hline \multirow{6}{*}{ 吕 } & $\mathrm{ADF}^{*}$ & 0 & $-3,903$ & $-3,654$ & $-2,957$ \\
\hline & $\mathrm{PP}^{*}$ & 4 & $-3,897$ & $-3,616$ & $-2,941$ \\
\hline & KPSS** & 4 & 0,397 & 0,739 & 0,463 \\
\hline & ERS PT & 4 & 9,049 & 1,990 & 3,260 \\
\hline & DFGLS & 4 & $-1,236$ & $-2,580$ & $-1,950$ \\
\hline & NG-Perron*** & 4 & $-1,1593$ & $-2,580$ & $-1,980$ \\
\hline
\end{tabular}

Fonte: Elaboração própria.

Nota: $\delta$ Defasagem minimiza o critério de Schwartz; " tabela MacKinnon (1996); ** tabela Kwiatkowski-Phillips-Schmidt-Shin (1992); **** tabela Perron (2001). 
Com o objetivo de se homogeneizar a comparação entre os diferentes testes e séries, se opta por utilizar, para os testes de raiz unitária, o nível de significância de $1 \%$, sabendo-se, no entanto, do problema de poder dos testes em amostras reduzidas e considerando que a amostra deste estudo possui apenas 40 observações. Assim, para a série de energia total (ver Tabela 2), em nível, nota-se que apenas o teste KPSS aponta para a não existência de uma raiz unitária, porém, ao se tomar a primeira diferençada variável, o único teste que aponta para a existência de raiz unitária é o ERS-PT. Dessa forma, há evidências de que a série do logaritmo da energia total seja I(1), uma vez que, tomando a primeira diferença, a série torna-se estacionária.

Para a série do PIB, quando analisada em nível, os resultados são ambíguos. Há evidências de que seja estacionária em torno de uma tendência determinística para os testes ADF, PP e ERSPT. De outro modo, os testes DFGLS, NG-Perron e KPSS apontam para existência de raiz unitária, este último a um nível de significância de $5 \% .^{3}$ Se analisadas as diferenças, todos os testes, exceto o DFGLS e o NG-Perron, apontam para inexistência de raiz unitária. Como há poucas evidências em modelos teóricos que justifiquem a possibilidade da variável PIB seguir um processo I(2), se assume a hipótese de que a série do logaritmo do PIB pode ser aproximada, como um processo não estacionário I(1).

A fim de se analisar os efeitos da renda das famílias e como essa está relacionada com o consumo de energia, outras duas séries serão analisadas em conjunto: a série do consumo de energia elétrica residencial e a série da renda bruta disponível, para o mesmo período (1970 a 2009). As Figuras 5 e 7 apresentam as séries em nível e as Figuras 6 e 8, em diferenças. Assim como para o PIB e o consumo total de energia, se realizam os mesmos testes para o consumo residencial e a renda bruta disponível.

Figura 5 - Log do Consumo de Energia Elétrica Residencial

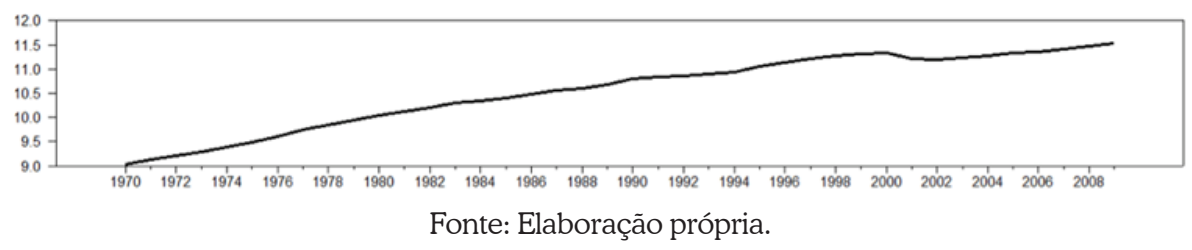

3 O teste KPSS também evidencia a presença de raiz unitária, porém a 5\% somente. 
Figura 6 - Primeira Diferença do Log do Consumo de Energia Elétrica Residencial

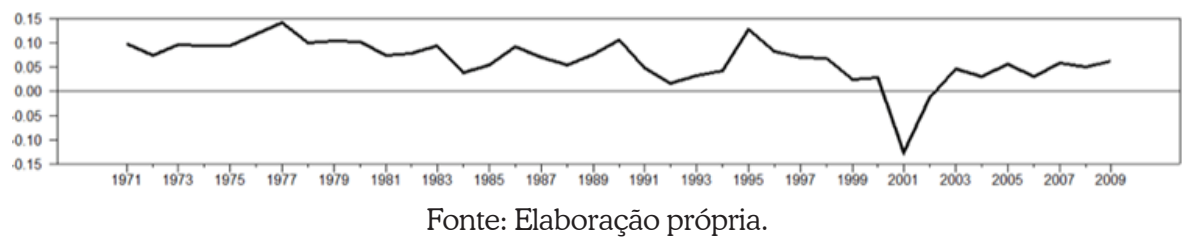

Figura 7 - Log da Renda Bruta Disponível

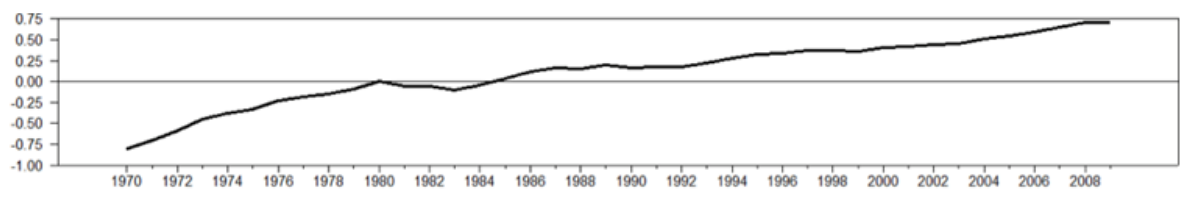

Fonte: Elaboração própria.

Figura 8 - Primeira Diferença do Log da Renda Bruta Disponível

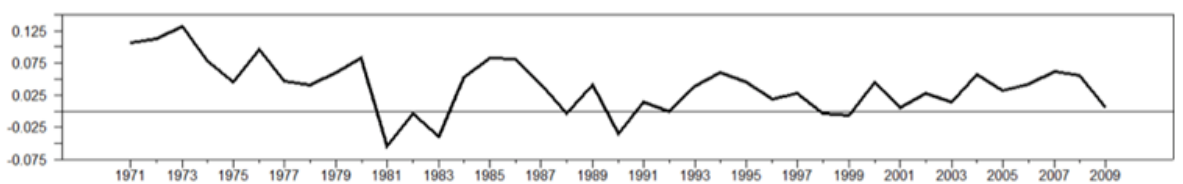

Fonte: Elaboração própria.

Nota-se que ambas as variáveis em nível apresentam uma tendência de crescimento, assim testa-se a hipótese de tendência estacionária. Quando examinadas em primeiras diferenças, nota-se que apresentam um comportamento estacionário, o que inicialmente nos leva a concluir que as séries são I(1). Assim, as Tabelas 4 e 5 apresentam os resultados na mesma estrutura já descrita para as séries de energia total e PIB, respectivamente. Observando os resultados para a série de energia elétrica, em ambos os casos, ou seja, tanto em nível, quanto em diferenças, há evidências de que haja raiz unitária. Isso, por sua vez, levaria a uma conclusão de que a série seria I(2), porém o gráfico da série em diferenças apresenta claramente um comportamento estacionário. 
Tabela 4 - Testes de raiz unitária para a série da energia elétrica residencial

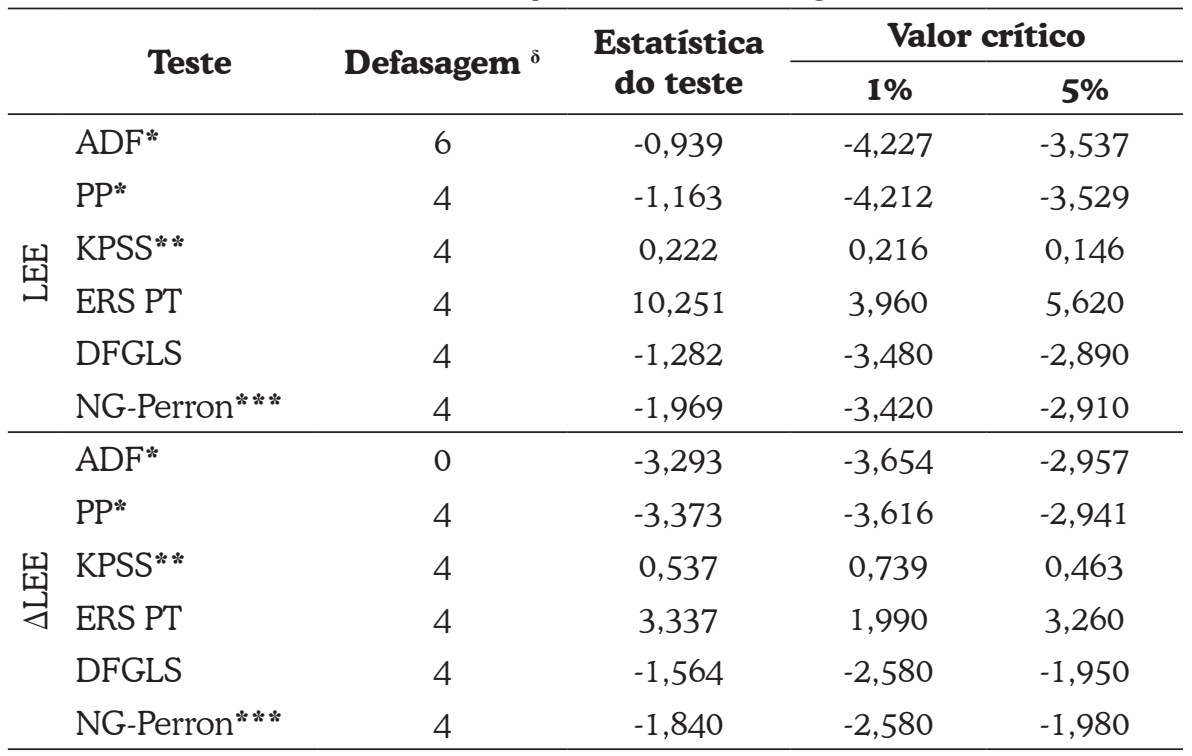

Fonte: Elaboração própria.

Nota: $\delta$ Defasagem minimiza o critério de Schwartz; * tabela MacKinnon (1996); ** tabela Kwiatkowski-Phillips-Schmidt-Shin (1992); *** tabela Perron (2001).

Tabela 5 - Testes de raiz unitária para a série da renda bruta disponível

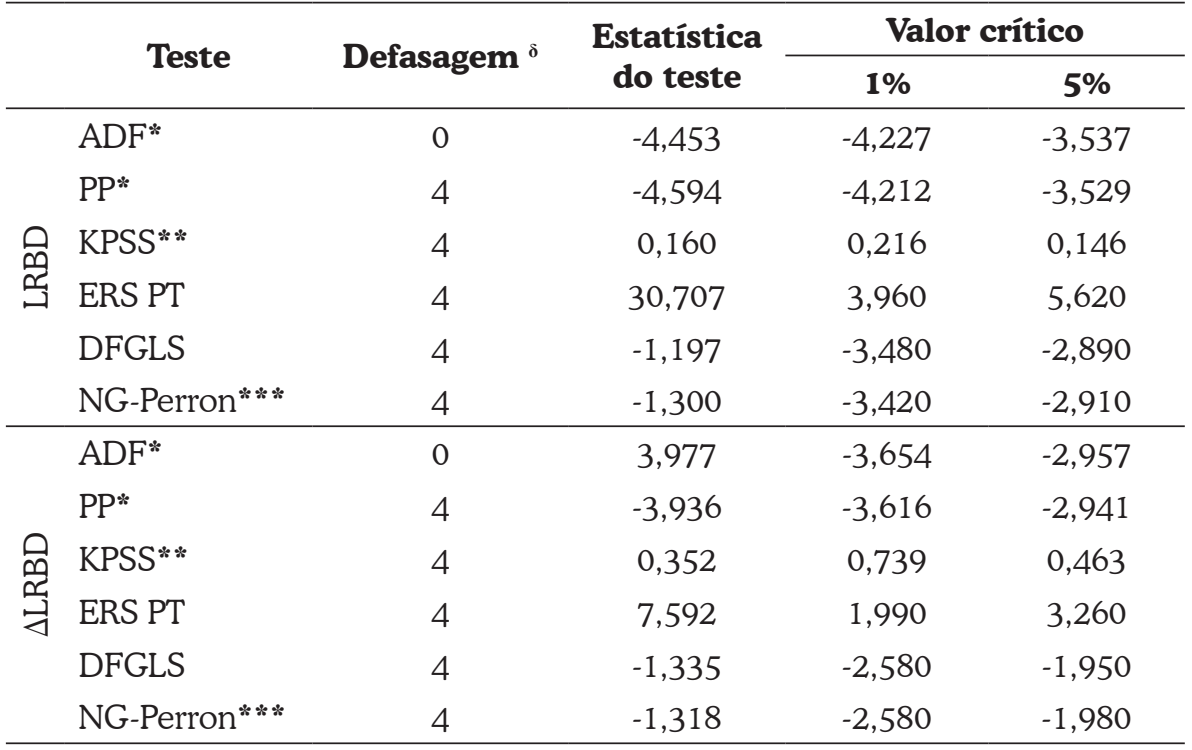

Fonte: Elaboração própria.

Nota: $\delta$ Defasagem minimiza o critério de Schwartz; * tabela MacKinnon (1996); "* tabela Kwiatkowski-Phillips-Schmidt-Shin (1992); *** tabela Perron (2001). 
Nota-se, entretanto, que, em 2001, há uma quebra na série, que representa o ano em que houve o racionamento de energia no Brasil devido a problemas no abastecimento nas usinas geradoras de energia. Perron (1989) afirma que as quebras estruturais na série levam à dificuldade de rejeitar a hipótese nula de raiz unitária, e, de forma empírica, isso parece ocorrer. Assim, como há evidências históricas, pois o racionamento possuiu um efeito evidente na série em diferenças, produzindo uma queda na taxa de crescimento significativa em 2001 (ver Figura 6), optou-se nesse caso por se realizar o teste de raiz unitária de Perron (1989). ${ }^{4}$ Esse teste incorpora uma quebra estrutural correspondendo a uma mudança no intercepto representado como o modelo A em Perron (1989). Adicionalmente, se realizou o teste proposto por Lee e Strazicich (2003), que considera a estimação dos pontos de quebra de maneira endógena.

Analisando, então, a série do logaritmo do consumo de energia elétrica pelas famílias em diferenças assumindo uma quebra estrutural em 2001, o resultado aponta para a rejeição ${ }^{5}$ da hipótese de raiz unitária. Da mesma maneira, o teste de Lee e Strazicich (2003) rejeita a hipótese nula de raiz unitária a 5\% para a série da primeira diferença do consumo residencial considerando o modelo com quebra no nível. Os resultados levam a concluir que a representação como uma série I(0) com uma quebra no intercepto seria mais adequada, e que, possivelmente, essa quebra pode estar causando um viés nos resultados dos testes de raiz unitária, no sentido de não rejeitar a hipótese nula. Logo, se conclui que a série do logaritmo do consumo de energia elétrica pelas famílias pode ser representada por um processo I(1).

Por sua vez, a série de renda bruta disponível (ver Tabela 5) se mostra não só visualmente similar à do PIB, como esperado, como também apresenta resultados dos testes de raiz unitária similares aos obtidos para o PIB. Apenas os testes DFGLS e NG-Perron apontam para a existência de raiz unitária para a série tanto em nível, quanto em primeiras diferenças. ${ }^{6}$ Desse modo, pelos mesmos motivos argumentados para a série do PIB, se assume que a renda bruta disponível pode ser aproximada por um processo I(1).

$4 \quad$ Fava e Cati (1995), analisando a série do PIB, apontam para a existência de quebras estruturais na economia brasileira em meados da década de 1980, porém, para o presente estudo, não houve problemas associados a não rejeição dos testes de raiz unitária que seriam resultado do viés imposto pela presença de quebras estruturais, como discutido em Perron (1989). Assim, optou-se pela não realização dos testes com quebra estruturais para as demais séries neste estudo.

5 O valor do teste de raiz unitária com base em Perron (1989) foi de -5,965, e o valor crítico a 1\% é de -3,750. Já para o teste Lee e Strazicich (2003), o resultado foi S $\{1\}=-0.7969$, com estatística $t=-4.4594$, e o valor crítico a $1 \%$ é de -4.54 e a $5 \%$ é de -3.84 . Os resultados em Lee e Strazicich são para uma amostra de tamanho $T=100$, enquanto a amostra efetiva deste trabalho é de 34 observações, o que se leva a ter cautela na interpretação dos resultados.

6 O teste KPSS também aponta presença de raiz unitária a 5\%. 
Com a caracterização de todas as séries estudadas como I(1), ${ }^{7}$ é possível, então, na próxima seção, realizar o trabalho com as mesmas, a fim de se obter um modelo que possa caracterizar o relacionamento entre elas, duas a duas, a saber: LET-LPIB e LEE-LRBD. Com esse modelo, questões amplamente discutidas, como relação de causalidade e impactos, poderão ser apresentadas de forma consistente.

\section{Modelos Empíricos}

Esta seção está focada na discussão dos modelos econométricos estimados. Num primeiro momento, é dada atenção ao caso do PIB e energia total, enquanto, num segundo momento, a atenção é voltada para o efeito renda (renda disponível e energia elétrica residencial).

Considerando-se a séries do PIB e energia total, os testes de determinação de defasagem realizados indicaram um VAR (8) para o critério HQ (Hanna-Quinn) e um VAR (1) para os critérios AIC (Akaike Information Criterion) e BIC (Bayesian Information Criterion). ${ }^{8}$ No entanto, os critérios AIC e BIC possuem tendência de escolher uma defasagem inferior à verdadeira em pequenas amostras, como discutido por Lütkepohl (2005). ${ }^{9}$

De outro modo, dado o reduzido tamanho da amostra, a estimação de um VAR (8) implicaria uma perda significativa de graus de liberdade. Por sua vez, os resultados dos testes de má especificação não indicaram a existência de não normalidade e autocorrelação nos resíduos quando se optou por um VAR(2). Desse modo, se escolheu essa ordem ao invés de um VAR(1). ${ }^{10}$ Como as variáveis são

$7 \quad$ Existe a possibilidade das séries de consumo serem integradas de ordem 2, I(2). Para reforçar os resultados, foram realizados os testes Dickey e Pantula (2002) nas séries de consumo de energia total e consumo de energia elétrica. Os resultados indicaram que, para o consumo residencial, existem evidências de que a série possa ser considerada I(2), contudo, se se considerar que o consumo de energia doméstica possui uma raiz unitária em primeira diferença, a implicação é que os choques existentes nessas variáveis teriam um caráter permanente no tempo. O impacto desses choques seria, na série em nível, o de aceleração no consumo de energia, dada a acumulação de choques permanentes, enquanto o que se observou para o consumo é um crescimento relativamente constante no tempo, tanto para o consumo total, quanto para o residencial, que apresentam uma média relativamente constante por mais de 38 anos. Cabe ressaltar, ainda, que, se os choques forem permanentes, a aceleração do consumo se daria num contexto de limitações impostas pelo crescimento da renda e da própria oferta de energia, não levando a crer que economicamente essa hipótese seja razoável para aproximar o processo gerador dos dados, e isso seria um resultado surpreendente. Ponderando as evidências e considerando que a hipótese da série possui duas raízes unitárias, em termos econômicos não seria incorreto supor que o processo possa ser bem aproximado como possuindo apenas uma raiz unitária num modelo com tendência para a série do consumo residencial.

8 Os resultados são omitidos por razões de espaço, mas podem ser obtidos com os autores.

9 O resultado obtido por Lütkepohl (2005), num experimento de Monte Carlo, é para um VAR bivariado com tamanho de amostra de 30 observações, portanto com estrutura e tamanho próximos à amostra deste trabalho, que totaliza 39 observações.

10 Os resultados dos testes foram: para autocorrelação de ordem 1: LM(1): ChiSqr(4) = 3.624 
assumidas I(1), utilizou-se a reparametrização na forma de um VECM (Vetor de Correção de Erros) com uma constante irrestrita consistente com uma tendência linear quando as variáveis são consideradas em nível. ${ }^{11} \mathrm{O}$ modelo é estimado por máxima verossimilhança, como proposto por Johansen (1996). Na Tabela 6 são apresentados os resultados do teste do traço.

Tabela 6 - Teste traço VAR Energia Total e PIB

\begin{tabular}{lll}
\hline $\mathbf{R}$ & $\mathbf{0}$ & $\mathbf{1}$ \\
\hline Trace Test & 19.913 & 0.940 \\
p-value & 0.009 & 0.332 \\
Corrected & 17.640 & 0.826 \\
p-value & 0.022 & 0.363 \\
Eigenvalue & 0.393 & 0.024 \\
\hline
\end{tabular}

Fonte: Elaboração própria.

Os resultados do teste indicam que é possível rejeitar a hipótese nula de que não há vetores de cointegração considerando-se um nível de significância de 5\%, já com o valor corrigido para pequenas amostras, como proposto por Johansen (2002), embora não haja rejeição a 1\%. Contudo, como o valor do módulo de uma das raízes da matriz companion do VAR possui valor muito próximo de 1 (0.977), e as demais apresentam valores menores do que 1 em módulo, se considerou que a existência de um vetor de cointegração e uma raiz unitária no sistema é uma aproximação razoável para o processo gerador dos dados..$^{12}$ Os resultados para a estimação dos vetores de cointegração são apresentados na Tabela 7.

[0.459]; autocorrelação de ordem 2: $\operatorname{LM}(2)$ : $\operatorname{ChiSqr}(4)=2.968$ [0.563]; normalidade: $\operatorname{ChiSqr}(4)$ = 3.836 [0.429]; efeitos ARCH: ordem (1) LM(1): ChiSqr(9) = 4.502 [0.875]; e ordem (2): LM(2): ChiSqr(18) $=4.849$ [0.999]. Os testes aplicados são descritos em detalhes por Cats in Rats (2005). O teste de autocorrelação é o teste LM de Godfrey, o teste de normalidade é uma variante do teste de Shenton e Bowman e o teste de efeitos ARCH multivariado segue o proposto por Lütkepohl e Krätzig (2004).

11 Uma discussão detalhada da representação em médias móveis de um VECM e do papel dos termos determinísticos pode ser encontrada no capítulo 6 de Juselius (2006). De maneira geral, um VECM sem constante é consistente com $E\left(\Delta Y Y_{t}\right)=0$ e $E\left(\beta^{\prime} Y\right)=0$, ou seja, taxas de crescimento nulas e vetores de cointegração com média zero. Já se for incluída uma constante irrestrita tem-se: $E(\Delta Y) \neq 0=\mu$ e $E\left(\beta^{\prime} Y\right) \neq 0$, ou seja, taxas de crescimento constantes em nível, aproximando um comportamento de tendência, como remete a análise das Figuras 1 e 3, e um vetor de cointegração com média diferente de zero. É preciso notar, ainda, que os Figuras 2 e 4 indicam que, em diferenças $(\Delta Y)$, as variáveis apresentam taxas de crescimento constantes com a série, sendo estacionárias em torno de uma média diferente de zero, o que reforça a escolha do VAR com uma constante irrestrita.

12 É preciso notar que o teste do traço na realidade é um teste para o número de raízes unitárias no sistema. Assim, a hipótese inicial é de $r=0$, ou seja, zero vetores de cointegração e duas raízes unitárias. Já para $r=1$ se tem uma raiz unitária e um vetor de cointegração, ou seja, as variáveis se combinam linearmente e formam uma nova variável estacionária. No caso de $r=2$, se tem um sistema com zero raízes unitárias e, portanto, estacionário em nível. Por sua vez, um VAR(p) pode 
Tabela 7 - Vetor de cointegração e coeficientes de ajustamento

\begin{tabular}{lll}
\hline Vetor de cointegração & $\begin{array}{l}\hat{\alpha}_{i} \\
\text { (se) }\end{array}$ & $\mathbf{i = 1}$ \\
\hline LPIB $=0.929$ LET & $\Delta$ LPIB & -0.228 \\
& & $(-3.275)$ \\
& $\Delta$ LET & -0.404 \\
& & $(-4.957)$ \\
Teste razão de verossimilhança para restrição & & \\
$\chi^{2}(1)=1.760[0.185]$ & & \\
\hline
\end{tabular}

Fonte: Elaboração própria.

Os resultados indicam que o vetor de cointegração é relevante nas duas equações, tanto do log do PIB, quanto do log do consumo de energia total. Ainda, o vetor de cointegração representa uma relação estacionária em que aumentos no PIB são consistentes com uma variação de mesma direção no consumo total de energia, de tal modo que a relação mantenha um comportamento estável de longo prazo. Isto é, as variáveis não se distanciam no longo prazo a despeito de possuírem um comportamento não estacionário quando tomadas individualmente.

O vetor de cointegração é significante e possui uma relação de correção de equilíbrio nas duas equações de tal modo que os desvios, tanto do PIB, quanto da energia total consumida da relação de longo prazo, tendem a se ajustarem para a relação de longo prazo expressa pelo vetor de cointegração. Em particular, se testou a hipótese de que o coeficiente da variável LET seja igual a 1, resultado que não pode ser rejeitado de acordo com o teste de razão de verossimilhança, já com a correção para pequenas amostras, portanto o vetor de cointegração pode ser reescrito como LPIB $=\beta$ LET.

ser escrito na sua forma companion como:

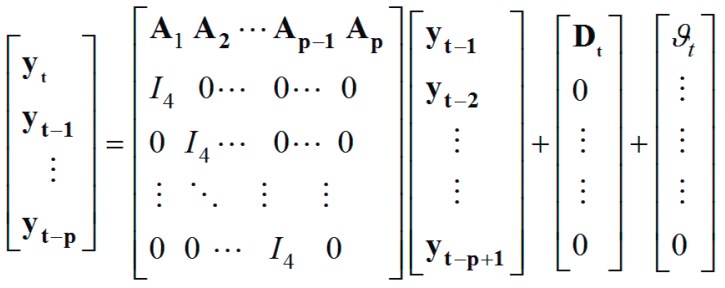

$$
\begin{aligned}
& \mathbf{Y}_{\mathbf{t}}=\Lambda \quad \mathbf{Y}_{\mathrm{t}-\mathbf{1}}+\mathrm{E}_{t}+\mathrm{T}_{t}
\end{aligned}
$$

Os autovalores da matriz companion são exatamente os autovalores de $\Lambda$. Nesse caso, se os autovalores estão dentro ou sobre o círculo unitário, então y é não estacionário, como sugere o módulo de uma das raízes indicando que o sistema possui uma raiz unitária. 
Quando foram considerados os testes de causalidade para ambas as formas de teste, $\mathrm{F}$ e chi-quadrado, ${ }^{13}$ os resultados (ver Tabela 8 ) indicaram que a hipótese de não causalidade do PIB para a energia total consumida não pode ser rejeitada a um nível de significância de 5\%, embora esse resultado seja apenas marginal. Já para a não causalidade da energia total consumida para o PIB, o teste indica claramente a rejeição, levando a crer, portanto, que a direção de causalidade parece ser do consumo de energia total para o PIB, embora a causalidade bidirecional não possa ser descartada.

Tabela 8 - Testes de causalidade PIB energia total consumida

\begin{tabular}{llll}
\hline $\begin{array}{l}\text { Direção } \\
\text { causalidade }\end{array}$ & $\begin{array}{l}\text { Tipo de teste } \\
\text { (graus de liberdade) }\end{array}$ & Estatística & p-value \\
\hline $\mathrm{PIB} \rightarrow \mathrm{ET}$ & $\mathrm{F}(2,30)$ & 2.9254 & 0.0691 \\
$\mathrm{PIB} \rightarrow \mathrm{ET}$ & $\chi^{2}(2)$ & 5.8507 & 0.0536 \\
$\mathrm{ET} \rightarrow \mathrm{PIB}$ & $\mathrm{F}(2,30)$ & 5.6809 & 0.0125 \\
$\mathrm{ET} \rightarrow \mathrm{PIB}$ & $\chi^{2}(2)$ & 10.1739 & 0.0062 \\
\hline
\end{tabular}

Fonte: Elaboração própria.

Considerando agora as séries da renda bruta disponível e do consumo de energia elétrica residencial, da mesma maneira que para as séries do PIB e energia total consumida, os testes de determinação da defasagem indicaram um VAR (8) para o critério HQ e um VAR (1) para os critérios AIC e BIC. Pelas mesmas razões apontadas anteriormente, se optou pela estimação de um VAR (2). Como as variáveis são assumidas I(1), se utilizou a reparametrização na forma de um VECM com uma constante irrestrita consistente com uma tendência linear quando as variáveis são consideradas em nível. Na Tabela 9 são apresentados os resultados do teste do traço.

13 É preciso notar que o teste realizado se refere a um teste de Wald em que a hipótese nula de não causalidade do PIB para energia total consumida é $\Gamma_{12}=\prod_{12}=0$ em: $\Delta \mathrm{Y}_{t}=\prod_{\mathrm{t}-1}+\Gamma_{1} \Delta \mathrm{Y}_{\mathrm{t}-1}+\epsilon_{\mathrm{t}}$, onde $\mathrm{Y}_{\mathrm{t}}=(\mathrm{LPIB}, \mathrm{LET})^{,}, \Pi=\left[\begin{array}{ll}\Pi_{11} & \Pi_{12} \\ \Pi_{21} & \Pi_{22}\end{array}\right], \quad \Gamma=\left[\begin{array}{ll}\Gamma_{11} & \Gamma_{12} \\ \Gamma_{21} & \Gamma_{22}\end{array}\right] \mathrm{e} \quad \epsilon_{t}=\left[\begin{array}{l}\epsilon_{1 t} \\ \epsilon_{2 t}\end{array}\right]$. Nesse caso, devido à hipótese do rank reduzido imposto pela existência de cointegração, a distribuição assintótica do teste convencional com base na estimação de um modelo com a restrição imposta não segue uma distribuição chi-quadrado. Lütkepohl (2005) propõe, então, um teste baseado em um VAR com defasagem aumentada, o qual corresponde ao teste apresentado na Tabela 8. 
Tabela 9 - Teste traço VAR energia residencial e renda bruta disponível

\begin{tabular}{lll}
\hline $\mathbf{R}$ & $\mathbf{0}$ & $\mathbf{1}$ \\
\hline Trace Test & 30.248 & 6.999 \\
-value & 0.000 & 0.008 \\
Corrected & 25.727 & 3.546 \\
-value & 0.001 & 0.060 \\
Eigenvalue & 0.548 & 0.168 \\
\hline
\end{tabular}

Fonte: Elaboração própria.

Similarmente ao ocorrido para o PIB e energia total consumida, os resultados do teste indicam a não rejeição da hipótese de $r=1$ vetores de cointegração apenas marginalmente. Contudo, como o valor do módulo de uma das raízes da matriz companion do VAR também possui valor muito próximo de 1 (0.961), e as demais apresentam valores menores do que 1 em módulo, se considerou que a existência de um vetor de cointegração e uma raiz unitária no sistema é uma aproximação razoável para o processo gerador dos dados. Os resultados para a estimação dos vetores de cointegração são apresentados na Tabela 10.

Tabela 10 - Vetor de cointegração e coeficientes de ajustamento

\begin{tabular}{lll}
\hline Vetor de cointegração & $\begin{array}{l}\hat{\alpha}_{i} \\
\text { (se) }\end{array}$ & $\mathbf{i = 1}$ \\
\hline LRBD=0.650LEE & $\Delta \mathrm{LRBD}$ & 0.051 \\
& & $(1.181)$ \\
& $\Delta \mathrm{LEE}$ & 0.122 \\
& & $(5.387)$ \\
Teste razão de verossimilhança para restrição & & \\
$\chi^{2}(1)=2.286$ [0.131] & & \\
\hline
\end{tabular}

Fonte: Elaboração própria.

Da mesma maneira que no modelo anterior, se testou a hipótese de que o coeficiente da variável LEE seja igual a 1, resultado que não pode ser rejeitado de acordo com o teste de razão de verossimilhança, já com a correção para pequenas amostras, portanto o vetor de cointegração pode ser reescrito como LRBD $=\beta L E E$

No entanto, diferentemente do ocorrido para o PIB e a energia total, os resultados indicam que o vetor de cointegração é relevante apenas na equação para o consumo de energia residencial. O vetor de cointegração representa uma relação estacionária em que aumentos na renda bruta disponível são consistentes com uma variação de mesma direção no consumo residencial de energia, de tal modo 
que a relação mantenha um comportamento estável de longo prazo, ou seja, as variáveis não se distanciam no longo prazo a despeito de possuírem um comportamento não estacionário quando tomadas individualmente.

Os desvios do consumo de energia residencial da relação de longo prazo, no entanto, não se ajustam para a relação de longo prazo expressa pelo vetor de cointegração. O coeficiente positivo $\alpha_{2}$ implica que, quando há choques positivos da renda de tal maneira que haja um excesso em relação ao consumo de energia elétrica consistente com o longo prazo expresso pelo vetor de cointegração, foram observados aumentos na taxa de crescimento do consumo residencial de energia.

Esse resultado parece consistente com o fato de que aumentos de renda disponível que desviem a variável do seu equilíbrio de longo prazo em relação a um consumo de energia elétrica residencial representam a maior disponibilidade de equipamentos eletroeletrônicos, que, por sua vez, induzem a maiores taxas de crescimento no consumo de energia residencial.

Por fim, são considerados os resultados dos testes de causalidade apresentados na Tabela 11. Para ambas as formas do teste, os resultados indicam que se pode rejeitar a hipótese de não causalidade da renda bruta para o consumo de energia residencial, enquanto que a não causalidade do consumo de energia residencial para a renda bruta disponível não pode ser rejeitada. A direção de causalidade, portanto, é bem definida da renda bruta disponível para o consumo residencial.

Tabela 11 - Testes de causalidade renda bruta disponível e consumo residencial

\begin{tabular}{llll}
\hline Direção causalidade & $\begin{array}{l}\text { Tipo de teste } \\
\text { (graus de liberdade) }\end{array}$ & Estatística & p-value \\
\hline $\mathrm{RBD} \rightarrow \mathrm{EE}$ & $\mathrm{F}(2,30)$ & 4.5424 & 0.0189 \\
$\mathrm{RBD} \rightarrow \mathrm{EE}$ & $\chi^{2}(2)$ & 9.0849 & 0.0106 \\
$\mathrm{EE} \rightarrow \mathrm{RBD}$ & $\mathrm{F}(2,30)$ & 0.2519 & 0.7789 \\
$\mathrm{EE} \rightarrow \mathrm{RBD}$ & $\chi^{2}(2)$ & 0.5038 & 0.7773 \\
\hline
\end{tabular}

Fonte: Elaboração própria.

\section{Considerações Finais}

O presente artigo buscou analisar a relação entre crescimento econômico (PIB) e consumo de energia no Brasil no período de 1970 a 2009. Tal análise foi realizada utilizando duas variáveis proxies para o crescimento econômico e duas para o consumo de energia, sendo utilizada a metodologia de séries temporais, que se mostra adequada para a análise a que se propõe este trabalho. É importante ressaltar, também, o tratamento dado à base de dados para minimizar os problemas que pudessem ocorrer devido ao tamanho da amostra. Foram realiza- 
dos vários testes de raízes unitárias que visaram justamente atender às questões inerentes ao poder dos testes e tamanho da amostra.

Em relação aos resultados é possível verificar que: a) a direção de causalidade parece ser do consumo de energia total para o PIB, embora a causalidade bidirecional não possa ser descartada; e b) considerando as variáveis de renda bruta disponível e consumo residencial de eletricidade, a direção de causalidade é bem definida da primeira para a segunda.

Um fato que pode levar a resultados conflitantes no que se refere aos resultados empíricos da relação entre consumo de energia e crescimento econômico está centrado na variedade de abordagens e nos procedimentos de testes empregados para se discutir tais questões.

O Quadro 1 apresenta um resumo dos resultados dos trabalhos para diversos grupos de países e diversas abordagens. Observando o quadro, é possível afirmar que os resultados para a economia brasileira vão ao encontro dos trabalhos empíricos. Mais especificamente, considerando-se a ocorrência de causalidade bidirecional em estudos para países em desenvolvimento (Paquistão, Filipinas, Tailândia, Coreia do Sul e Argentina), é possível assumir que esteja presente também no caso brasileiro, uma vez que a não rejeição da hipótese de não causalidade da energia total consumida para o PIB é marginal.

Quadro 1 - Resultados da causalidade entre crescimento e consumo de energia para uma amostra de países

\begin{tabular}{|c|c|c|}
\hline Autor & Amostra de países & $\begin{array}{l}\text { Direção de } \\
\text { causalidade }\end{array}$ \\
\hline $\begin{array}{c}\text { Kraft e Kraft } \\
\text { (1978). }\end{array}$ & Estados Unidos. & $\mathrm{PIB} \rightarrow \mathrm{CE}$ \\
\hline $\begin{array}{c}\text { Akarca e Long } \\
\text { (1979). }\end{array}$ & Estados Unidos. & $\mathrm{CE} \rightarrow$ emprego \\
\hline $\begin{array}{l}\text { Masih e Masih } \\
\text { (1996). }\end{array}$ & $\begin{array}{l}\text { Índia. } \\
\text { Paquistão. } \\
\text { Indonésia. } \\
\text { Malásia, Singapura e Filipinas. }\end{array}$ & $\begin{array}{l}\mathrm{CE} \rightarrow \mathrm{PIB} \\
\mathrm{PIB} \rightarrow \mathrm{CE} \\
\mathrm{PIB} \rightarrow \mathrm{CE} \\
\text { Neutro } \\
\end{array}$ \\
\hline $\begin{array}{c}\text { Glasure e Lee } \\
\text { (1998). }\end{array}$ & Coreia do Sul e Cingapura & $\mathrm{PIB} \rightarrow \mathrm{CE}$ \\
\hline $\begin{array}{l}\text { Asafu-Adjaye } \\
\text { (2000). }\end{array}$ & $\begin{array}{l}\text { Índia e Indonésia. } \\
\text { Filipinas e Tailândia. }\end{array}$ & $\begin{array}{l}\mathrm{CE} \rightarrow \mathrm{PIB} \\
\mathrm{PIB} \rightarrow \mathrm{CE}\end{array}$ \\
\hline
\end{tabular}

continua... 
conclusão.

\begin{tabular}{|c|c|c|}
\hline Autor & Amostra de países & $\begin{array}{l}\text { Direção de } \\
\text { causalidade }\end{array}$ \\
\hline $\begin{array}{l}\text { Soytas e Sari } \\
\quad(2003)\end{array}$ & $\begin{array}{l}\text { Argentina. } \\
\text { Itália e Coreia do Sul. } \\
\text { Turquia, França, Alemanha e Japão. } \\
\text { Brasil, Índia, Indonésia, México, } \\
\text { Polônia, África do Sul, Estados Unidos, } \\
\text { Reino Unido e Canadá. }\end{array}$ & $\begin{array}{l}\mathrm{PIB} \rightarrow \mathrm{CE} \\
\mathrm{PIB} \rightarrow \mathrm{CE} \\
\mathrm{CE} \rightarrow \mathrm{PIB} \\
\\
\text { Neutro }\end{array}$ \\
\hline $\begin{array}{c}\text { Altinay e Karagol } \\
(2004) \text {. }\end{array}$ & Turquia. & Neutro \\
\hline $\begin{array}{l}\text { Oh e Lee }(2004 a \\
2004 b) .\end{array}$ & Coreia do Sul. & $\mathrm{PIB} \rightarrow \mathrm{CE}$ \\
\hline $\begin{array}{c}\text { Chontanawat et al. } \\
\text { (2008). }\end{array}$ & $\begin{array}{l}\text { Países da OECD ( } 76 \%) \text {. } \\
\text { Países não membros da OECD (40\%). }\end{array}$ & $\begin{array}{l}\mathrm{PIB} \rightarrow \mathrm{CE} \\
\mathrm{PIB} \rightarrow \mathrm{CE}\end{array}$ \\
\hline $\begin{array}{l}\text { Huang, Hwang e } \\
\text { Yang (2008). }\end{array}$ & $\begin{array}{l}\text { Países de alta renda. } \\
\text { Países de média-alta renda. } \\
\text { Países de média-baixa renda. } \\
\text { Países de baixa renda. }\end{array}$ & $\begin{array}{l}\mathrm{CE} \rightarrow \mathrm{PIB} \\
\mathrm{PIB} \rightarrow \mathrm{CE} \\
\mathrm{PIB} \rightarrow \mathrm{VE} \\
\text { Neutro }\end{array}$ \\
\hline
\end{tabular}

Fonte: Elaboração própria.

Dessa forma, em termos de políticas públicas, o resultado apresentado evidencia que, caso houvesse uma diminuição na demanda por energia, haveria uma queda no crescimento econômico. Extrapolando, pode-se inferir que, caso um choque de oferta de energia ocorresse, fazendo com que a demanda diminua, o resultado seria o mesmo. Entretanto, não é possível afirmar que, caso houvesse uma diminuição do crescimento econômico, haveria uma queda na demanda por energia, porém esse resultado não pode ser descartado.

Assim, deve-se ter em mente que, ao atender ou estimular a demanda por energia das empresas e famílias, o crescimento da economia acompanhará, ceteris paribus, essa trajetória. Isso pode implicar a necessidade de outros insumos para atender à demanda maior como um todo da economia, isto é, pode haver um efeito multiplicador na economia, gerando maiores fluxos de renda e emprego.

Em relação ao resultado de causalidade entre renda bruta disponível e consumo de energia, há uma série de considerações que podem ser realizadas. Em primeiro lugar, há que se considerar que o processo de crescimento da economia brasileira, e, por consequência, o aumento de renda da população brasileira, principalmente das classes $\mathrm{C} \mathrm{e} \mathrm{D}$, apontam para a necessidade de se levar em consideração esse processo na formulação de políticas para o setor. As conclusões deste estudo, quando levado em conta o estabelecimento claro de uma direção de causalidade num contínuo de tempo de quase 40 anos, indicam que há de se conside- 
rar a necessidade de inclusão dessa variável como indicador de políticas de longo prazo para o setor. Em segundo lugar, a discussão sobre eficiência energética (por exemplo, o caso da Procel, entre outros) deve estar presente nos debates sobre o tema, uma vez que as limitações de oferta para esse setor não são superadas no curto prazo. Os resultados indicam claramente que desequilíbrios existentes na relação de longo prazo, representada por aumentos na renda bruta disponível, que a distancie do consumo de energia residencial, tem o efeito de acelerar o consumo de energia residencial no curto prazo - representado pelo sinal positivo do coeficiente do vetor de cointegração apresentado na Tabela 10 .

\section{Referências}

AKARCA, A. T.; LONG, T. V. Energy and employment: a time series analysis of the causal relationship. Resources Energy, v. 2, n. 2-3, p. 151-162, 1979.

ALTINAY G.; KARAGOL, E. Structural break, unit root, and the causality between energy consumption and GDP in Turkey. Energy Economics, v. 26, n. 6, p. 985-994, 2004.

ASAFU-ADJAYE, J. The relationship between energy consumption, energy prices and economic growth: time series evidence from Asian developing countries. Energy Economics, v. 22, n. 6 , p. 615-625, 2000.

CANOVA, F. The economics of VAR models. In: HOOVER, K. D. (Org.). Macroeconometrics: developments, tensions and prospects. Boston: Kluwer Academic Publishers, 1995.

CATS IN RATS. Versão 2. Evanston, IL: Estima, 2005. 1 CD-ROM.

CHONTANAWAT, J.; HUNT, L.; PIERSE, R., Does energy consumption cause economic growth?: Evidence from a systematic study of over 100 countries. Journal of Policy Modeling, v. 30, n. 2, p. 209-220, 2008.

DICKEY, D. A.; FULLER, W. A. Distribution of the estimators for autoregressive time series with a unit root. Journal of the American Statistical Association, v. 74, n. 366, p. 427-431, 1979.

DICKEY, D. A.; FULLER, W. A. Likelihood Ratio Statistics for Autoregressive Time Series with a Unit Root. Econometrica, v. 49, n. 4, p. 1057-72, 1981.

DICKEY, D.; PANTULA, S. G. Determining the order of differencing in autoregressive processes. Journal of Business 8 Economic Statistics, v. 20, n. 1, p. 18-24, 2002. Twentieth Anniversary Commemorative Issue.

ELLIOT, G.; ROTHENBERG, T. J.; STOCK, J. H. Efficient tests for an autoregressive unit root. Econometrica, v. 64, n. 4, p. 813-836, 1996.

EMPRESA DE PESQUISA ENERGÉTICA. Balanço Energético Nacional 2010: ano base 2009. Rio de Janeiro: EPE, 2010.

FAVA, V. L; CATI, R. C. Mudanças no comportamento do PIB brasileiro: uma abordagem econométrica. Pesquisa e planejamento econômico, v. 25, n. 2, p. 279-296, 1995. 
GHALI, K. H.; EL-SAKKA, M. I. T. Energy use and output growth in Canada: a multivariate cointegration analysis. Energy Economics, v. 26, n. 2, p. 225-238, 2004.

GLASURE, Y. U. Energy and national income in Korea: further evidence on the role of omitted variables. Energy Economics, v. 24, n. 4, p. 355-365, 2002.

GLASURE, Y. U.; LEE, A-R. Cointegration, error-correction and the relationship between GDP and energy: the case of South Korea and Singapore. Resources and Energy Economics, v. 20, n. 1, p. 17-25, 1998.

GRANGER, C.; NEWBOLD, P. Spurious regressions in econometrics. Journal of. Econometrics, v. 2, n. 2, p. 111-120, 1974.

HAMILTON, J. D. Time series analysis. Princeton: Princeton University Press, 1994.

HENDRY, D. F. Dynamic econometrics. Oxford: Oxford University Press, 1995. .

HONDROYIANNIS, G.; LOLOS, S.; PAPAPETROU, E. Energy consumption and economic growth: assessing the evidence from Greece. Energy Economics, v. 24, n. 4, p. 319-336, 2002.

HUANG, B. N.; HWANG, M. J.; YANG, C. W. Causal relationship between energy consumption and GDP growth revisited: a dynamic panel data approach. Ecological Economics, v. 67, n. 1, p. 41-54, 2008

IBGE. Contas nacionais. Disponível em: <http://downloads.ibge.gov.br/downloads estatisticas.htm >. Acesso em: 15 out. 2012.

IPEADATA. Disponível em <http://www.ipeadata.gov.br> . Acesso em: 15 out. 2012.

JOHANSEN, S. A small sample correction for the test of cointegrating rank in the vector autoregressive model. Econometrica, v. 70, n. 5, p. 1929-1961, 2002.

. Likelihood-based inference in cointegrated vector autoregressive models. Oxford: Oxford University Press, 1996.

JUSELIUS, K. The cointegrated VAR model: methodology and applications. New York: Oxford University Press, 2006.

KRAFT, J.; KRAFT, A. On the relationship between energy and GNP. Journal of Energy and Development, v. 3, n. 2, p. 401-403, 1978.

KWIATKOWSKI, D. et al. Testing the null hypothesis of stationary against the alternative of a unit root. Journal of Econometrics, v. 54, n. 1-3, p. 159-178, 1992.

LEE, J.; STRAZICICH, M. C. Minimum Lagrange multiplier unit root test with two structural breaks. The Review of Economics and Statistics, v. 85, n. 4, p. 1082-1089, 2003.

LÜTKEPHOL, H.; KRÄTZIG, M. (Ed.). Applied Time Series Econometrics, Cambridge: University Press, Cambridge, 2004.

LÜTKEPOHL, H. New introduction to multiple time series analysis. Heidelberg: SpringerVerlag, 2005. 
MACKINNON, J. G. Numerical distribution functions for unit root and cointegration tests. Journal of Applied Econometrics, v. 11, n. 6, p. 601-618, 1996.

MADDALA, G. S.; KIM, I. M. Unit roots, cointegration and structural change. Cambridge: Cambridge University Press, 1998.

MASIH, A. M. M.; MASIH, R. Energy consumption, real income and temporal causality: results from a multi-country study based on cointegration and error-correction modeling techniques. Energy Economics, v. 18, n. 3, p. 165-183, 1996.

NELSON, C. R.; PLOSSER, C. I. Trends and random walks in macroeconomic time series. Journal of Monetary Economics, v. 10, n. 2, p. 139-162, 1982.

NG, S.; PERRON, P. Lag Length Selection and the Construction of Unit Root Tests with Good Size and Power. Econometrica, v. 69, n. 6, p. 1519-1554, 2001.

$\mathrm{OH}, \mathrm{W}$; LEE, K. Causal relationship between energy consumption and GDP revisited: the case of Korea 1970-1999. Energy Economics, v. 26, n. 1, p. 51-59, 2004 a.

. Energy consumption and economic growth in Korea: testing the causality relation.

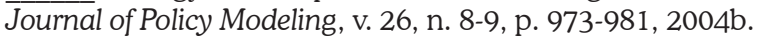

PÊGO FILHO, B. et al. Impactos fiscais da crise de energia elétrica: 2001 e 2002. Rio de Janeiro: Ipea, 2001. (Texto para discussão, n. 816).

PERRON, P. The great crash, the oil price shock and the unit root hypothesis, Econometrica, v. 57, n. 6, p. 1361-1401, 1989.

SARI, R.; SOYTAS, U. Disaggregate energy consumption, employment, and income in Turkey. Energy Economics, v. 26, n. 3, p. 335-344, 2004.

SCHMIDT, C. A. J.; LIMA, M. A .M. A demanda por energia elétrica no Brasil. Revista Brasileira de Economia, v. 58, n. 1, p. 67-98, 2004.

SIMS, C. A. Macroeconomics and reality. Econometrica, v. 48, n. 1, p. 1-48, 1980.

SOYTAS, U.; SARI, R. Energy consumption and GDP: causality relationship in G-7 countries and emerging markets. Energy Economics, v. 25, n. 1, p. 33-37, 2003.

SOYTAS, U.; SARI, R. The relationship between energy and production: Evidence from Turkish manufacturing industry. Energy Economics, v. 29, n. 6, p. 1151-116, 2007.

STERN, D. I. A multivariate cointegratioin analysis of the role of energy in the US macroeconomy. Energy Economics, v. 22, n. 2, p. 267-283, 2000.

STERN, D. I. Limits to substitution and irreversibility in production and consumption: a neoclassical interpretation of ecological economics. Ecological Economics, v. 21, n. 3, p. 197215, 1997.

YANG, H. Y. A note on the causal relationship between energy and GDP in Taiwan. Energy Economics, v. 22, n. 3, p. 309-317, 2000. 
Recebido em: 15/01/2014. Aceito em: 19/09/2014. 